\title{
Moisture-Responsive Polymer Films on Flexible Substrates for Optical Sensing of Humidity ${ }^{\dagger}$
}

\author{
Katerina Lazarova ${ }^{1, *}$, Silvia Bozhilova ${ }^{2}$, Sijka Ivanova ${ }^{2}$, Darinka Christova ${ }^{2}$ and \\ Tsvetanka Babeva ${ }^{1, *}$ \\ 1 Institute of Optical Materials and Technologies “Acad. J. Malinowski”, Bulgarian Academy of Sciences, \\ Akad. G. Bonchev str., bl. 109, 1113 Sofia, Bulgaria \\ 2 Institute of Polymers, Bulgarian Academy of Sciences, Akad. G. Bonchev Str., bl. 103-A, 1113 Sofia, \\ Bulgaria \\ * Correspondence: klazarova@iomt.bas.bg (K.L.); babeva@iomt.bas.bg (T.B.); Tel.: +359 02-979-3521 (K.L.) \\ + Correspondence:Presented at the 7th International Electronic Conference on Sensors and Applications, 15- \\ 30 November 2020; Available online: https://ecsa-7.sciforum.net/.
}

Published: 15 November 2020

\begin{abstract}
In this paper the possibility to design flexible humidity sensors by spin-coating of moisture-sensitive polymer on three types of substrates: poly(ethylene terephthalate) (PET), polylactide (PLA) and composite polysiloxane is investigated. The optical properties, surface morphology and roughness of the substrates covered with polymer are studied by transmittance measurements and surface profiling, respectively. Thin polymer films of amphiphilic copolymer obtained by partial acetalization of poly(vinyl alcohol) are used as humidity sensitive media. The sensing properties are probed through transmittance measurements at different levels of relative humidity (RH). The influence of substrate type is studied by comparing hysteresis of flexible sensors with those deposited on glass substrates.
\end{abstract}

Keywords: humidity; optical sensor; polymers; flexible substrates; thin films

\section{Introduction}

Depending on the application and the corresponding requirements, various techniques have been applied for humidity measurements and different types of sensors have been developed. Traditional humidity sensors are based on the electrical measurement. Optical sensing of humidity, where detection relies on change of optical properties such as refractive index, optical thickness, color, etc., emerges as an alternative approach [1,2] due to the offered advantages like operation at room temperature, high accuracy, resistance to electromagnetic interference and lack of explosion danger. Development of biosensors for in situ monitoring of biological fluids, wearable electronics and flexible/stretchable sensors for gaseous analytes in the surrounding environment, that affects human health, are of particular interest [3-6]. Developing such sensors faces multiple challenges: compatible materials, suitable manufacturing techniques, suitable substrates, readout interface, etc. Recent review [7] shows that most optical sensors are optical fibers type for flexible temperature sensors and as included in humidity sensors, flexible UV-Exposure optical devices wrapped around fingers for monitoring skin health [8] and more. It would be a novelty to make sensors on a flexible substrate that do not require power supply, have easy production technology and rely entirely on optical detection by simple change of the color. To our knowledge there is no data on experiments to obtain such a sensor. Great variety of materials can be used that change their refractive index, extinction coefficient or thicknesses as a sensitive media deposited on flexible substrates [9-11]. And polymers are materials that stand out because of their easy deposition in form of thin films and relatively low cost, tailored functionality and fast response owing to short diffusion path length [12]. 
In our previous studies $[13,14]$ we have shown that thin films of poly(vinyl alcohol-co-vinyl acetal)s (PVA-Ac) with acetal content in the range 18-28\%, which are hydrophobically modified PVA copolymers, are suitable for optical sensing of humidity in both - transmission and reflection mode, depending on the substrate. The best sensor characteristics are obtained for PVA-Ac with acetal content $24 \%$ at film thickness of $80 \mathrm{~nm}$ and post-deposition annealing temperature of $60{ }^{\circ} \mathrm{C}$. Measuring in transmittance regime is easier, more accurate, and less expensive than measuring in reflectance mode, therefore it will be more advantageous to use substrates that are transparent and allow transmittance measurements for detecting humidity. Flexible substrates, as well as glass or plastic have an approximate refractive index in the range 1.4-1.5 that is very close to the refractive index of the humidity sensitive polymers used for detection. To overcome the small optical contrast, that will lead to low sensitivity of detection, thin sublayer can be deposited between the substrate and the sensitive media.

In this study we use three types of substrates-poly(ethylene terephthalate) (PET), polylactide (PLA) and composite polysiloxane (Compo-Sil by General silicones Co, Ltd). The substrates were coated with a single $30 \mathrm{~nm}$ Au:Pd layer before spin-coating the modified PVA copolymer with acetal content $24 \%$ (PVA-Ac24). The influence of the type of substrate on the optical characteristics and humidity sensing properties of the active polymer thin films was investigated.

\section{Materials and Methods}

Hydrophobically modified PVA copolymer, namely PVA-Ac24 was synthesized by partial acetalization of hydroxyl groups of PVA with acetaldehyde as already described [13-15]. For the thin film deposition, copolymer solutions of concentration $1 \mathrm{wt}$. \% in mixed water-methanol solvent (20:80 volume ratio) were prepared.

PET, PLA and Compo-Sil (composite polysiloxane by General silicones Co, Ltd) flexible substrates as well as borosilicate glass were covered with Au:Pd sublayer with gold-palladium ratio of 80:20 and thickness of $30 \mathrm{~nm}$. Thin metal films were deposited on all substrates by cathode sputtering of gold/palladium target (Quorum Technologies, Lewes, UK) for $60 \mathrm{~s}$ under vacuum $4 \times$ $10^{-2}$ mbar by using Mini Sputter Coater SC7620 system (Quorum Technologies, Lewes, UK). For the purpose of determining the thickness of the polymer film a silicon wafer/substrate ( $\mathrm{Si}$ ) was also used for the deposition of a single film.

Thin polymer films were deposited on all transparent substrates with metal sublayer and on $\mathrm{Si}$ wafer via spin-coating method by dropping $0.250 \mathrm{~mL}$ of the solution on the substrate and then spun stepwisely. In the first step with duration of $1.5 \mathrm{~s}$ the substrate is rotated at $2500 \mathrm{rpms}$. The second stage is carried out at $4000 \mathrm{rpm}$ for $60 \mathrm{~s}$. In both steps the acceleration speed stays $2500 \mathrm{rpm} / \mathrm{s}$. Post annealing process in air took place afterwards for $30 \mathrm{~min}$ at $60{ }^{\circ} \mathrm{C}$. For the monitoring of the surface roughness of deposited films 3D Optical profiler (Zeta-20, Zeta Instruments) was used.

Thus obtained thin polymer films have thickness $80 \mathrm{~nm}$, calculated from measured reflectance spectra with UV-VIS-NIR spectrophotometer (Cary 5E, Varian) by using previously developed twostage nonlinear curve fitting method [16]. Same method was used in order to calculate the optical constant $n$ (refractive index) and $k$ (extinction coefficient) of the PVA-Ac24 film. Sensing properties of the films were studied in transmittance mode $(\mathrm{T})$ by measuring spectra in humidity range from 5 to $95 \% \mathrm{RH}$ at fixed wavelength that is preliminary chosen as the wavelength of the highest humidity responses (transmittance change $\left.\Delta \mathrm{T}_{\max }=\mid \mathrm{T}_{95 \mathrm{RH}}-\mathrm{T}_{5 \mathrm{RH}} \mathrm{I}\right)$. To conduct humidity sensing experiments was used a homemade bubbler system that generated vapors from liquids and cell with a humidity sensor integrated in it [17].

In order to study the hysteresis that is express as different values of $\mathrm{T}$ measured at the same values of humidity depending whether humidity increases or decreases, the percentage of hysteresis (H) was defined by:

$$
H(\%)=\frac{\max \left|T_{u p-} T_{\text {down }}\right|}{\Delta T_{\max }} \cdot \frac{\Delta R H_{\text {hyst }}}{\Delta R H} \cdot 100
$$


where $T_{u p}$ and $T_{\text {down }}$ are transmittance values measured for increasing and decreasing humidity, respectively, $\Delta T_{\max }$ is the transmittance change in the whole range $\Delta R H$ of measured humidity and $\Delta R H_{\text {hyst }}$ is the humidity range where hysteresis is observed.

\section{Results and Discussion}

\subsection{Optical Properties}

First step in studying the optical properties of flexible substrates was to measure their transmittance in UV-visible range $-320-800 \mathrm{~nm}$ before depositing any thin films. Figure 1a shows transmittance spectra of PET, PLA, Compo-Sil flexible substrates and borosilicate glass. Spectra of PET and PLA samples have almost identical T spectra and transmittance value of $87 \%$ at $600 \mathrm{~nm}$ which is very close to transmittance value of $91 \%$ for borosilicate glass. Compo-Sil substrate have lower transparency level and transmittance coefficient $-77 \%$. One of the advantages of Compo-Sil, although its lower transparency, is that this material is more stretchable than PET and PLA.

Next step was the deposition of the Au:Pd/PVA-Ac24 films on the flexible substrates and glass, after which transmission spectra were measured. Refractive index $n$ and extinction coefficient $k$ values of thin films of PVA-Ac24 with thickness about $80 \mathrm{~nm}$ were previously calculated [18] and taken at wavelength of $600 \mathrm{~nm}-1.45$ and 0.017 , respectively, from measured reflectance spectra of the polymer deposited on silicon wafer. As seen on Figure $1 \mathrm{~b}$ the spectra of PET covered with $\mathrm{Au}: \mathrm{Pd} / \mathrm{PVA}-\mathrm{Ac} 24$ is very close to the spectra of the same structure deposited on glass. The transmission coefficient at $600 \mathrm{~nm}$ wavelength is $55 \%$ for PET and $59 \%$ for glass. The other twoCompo-Sil and PLA-have lower transparency level and transmission coefficients $44 \%$ and $36 \%$, respectively. All samples are transparent in the visible range.
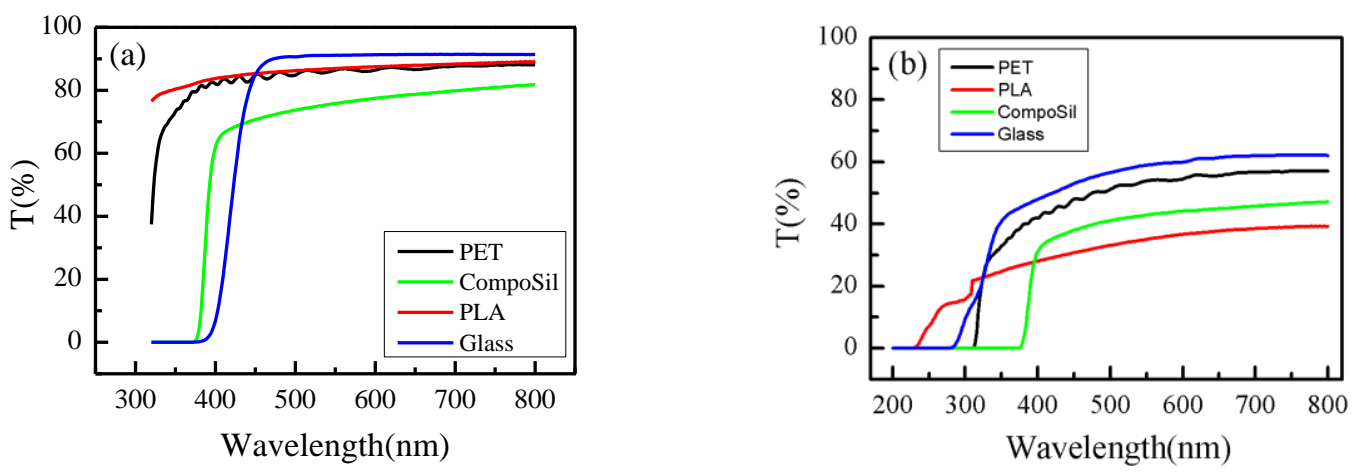

Figure 1. Transmittance spectra of bare (a) and covered with Au:Pd/PVA-Ac24 film (b) substrates: glass, PET, PLA and Compo-Sil.

\subsection{Surface Morphology}

Examination of the surface of the samples with a 3D Optical profiler confirmed the transmission coefficient results discussed above. The pictures shown in Figure 2a clearly indicate that PET substrate with $\mathrm{Au}: \mathrm{Pd} /$ polymer deposited on top has the smoothest surface and even distribution of the thickness, followed by the Compo-Sil sample. It is seen that PLA sample is inhomogeneous and with different thickness sections (different colors on the picture), which is a prerequisite for the stronger scattering and lower light transmission. These differences may be due to the adhesion of every substrate itself and the different film formation during the spin coating process. Another possible reason is the heat treatment after the deposition of the thin films that could decrease the mechanical strength of the substrates especially those of PLA that is the thinnest one. 

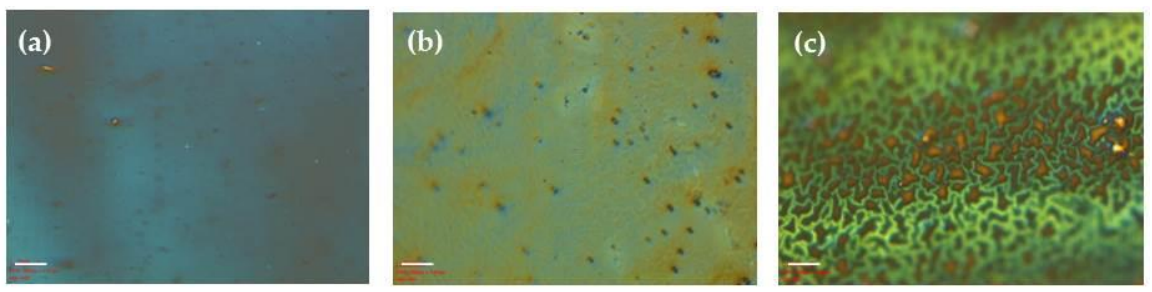

Figure 2. Pictures of surfaces of PET (a), Compo-Sil (b) and PLA (c) substrates with deposited $\mathrm{Au}: \mathrm{Pd} / \mathrm{PVA}-\mathrm{Ac} 24$ at a magnification of 50x (the bar is $15 \mu \mathrm{m}$ ).

\subsection{Sensing Properties}

In order to study the sensing ability of samples towards moisture/humidity, transmission spectra were measured at low and high relative humidity levels ( $5 \% \mathrm{RH}$ and $95 \% \mathrm{RH})$. As it is seen from Figure 3a, that shows the change of the measured spectra of PET substrate with deposited Au:Pd/PVA-Ac24, when exposed to low and high relative humidity, the highest humidity response $\Delta T_{\max }$ of $5.9 \%$ is achieved at wavelength of $476 \mathrm{~nm}$.
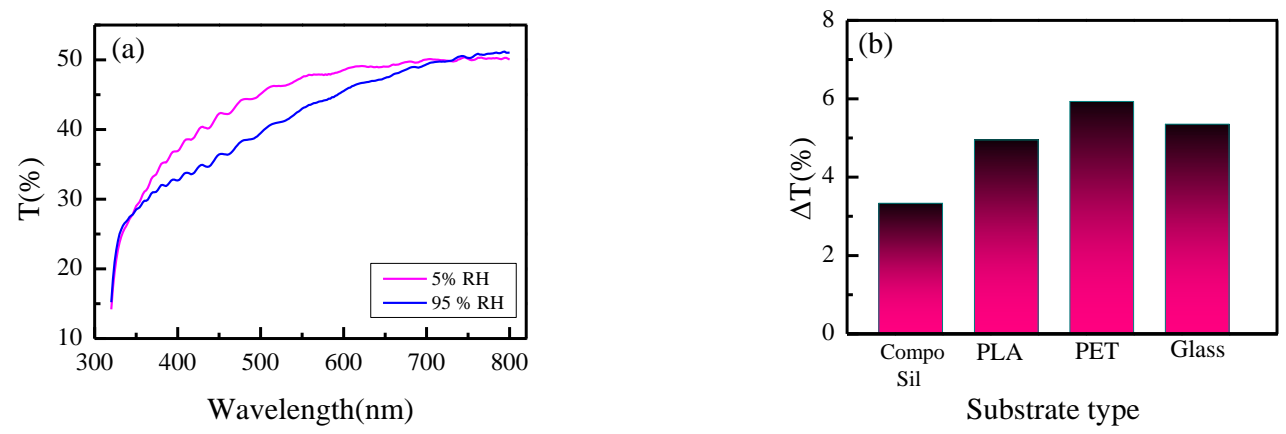

Figure 3. (a) Measured transmission spectra of PET substrate with deposited Au:Pd/PVA-Ac24 when exposed to low (magenta) and high (blue) relative humidity; (b) Transmittance change, $\Delta T_{\max }(\%)$ calculated from measured transmission spectra of Compo-Sil, PLA, PET and glass substrates with deposited Au:Pd/PVA-Ac24 at low and high relative humidity.

Same measurements were conducted with other two flexible substrates and with glass sample as well. Calculated values of transmittance change $\Delta T_{\max }$ of all samples are shown on Figure $3 \mathrm{~b}$. The value of transmittance change $\Delta T_{\max }$ for Compo-Sil sample is the smallest (3.3\%) compared to other substrates and the strongest response is achieved for PET sample (5.9\%). Again, this result can be explained with surface roughness and transparency of the substrates as well as with different adhesion of film to different substrates.

Although the glass showed better transparency, the reaction of PET substrate is stronger compared to the glass and in this sense, we observe a small improvement in the sensing properties of the polymer film deposited on flexible substrate as regards to the glass substrate. But this is not the only indicator by which we evaluate the sensing properties.

A very important parameter that determines the suitability of the material for sensing applications, as it is well known, is the hysteresis, $\mathrm{H}$. If $\mathrm{H}$ exists that means that for the same humidity values, depending whether humidity increases or decreases, different transmittance value occurs. To keep its value as low as possible is our main goal. The hysteresis values $\mathrm{H}$ of all flexible substrates along with glass sample are calculated and summarized in Figure 4. 


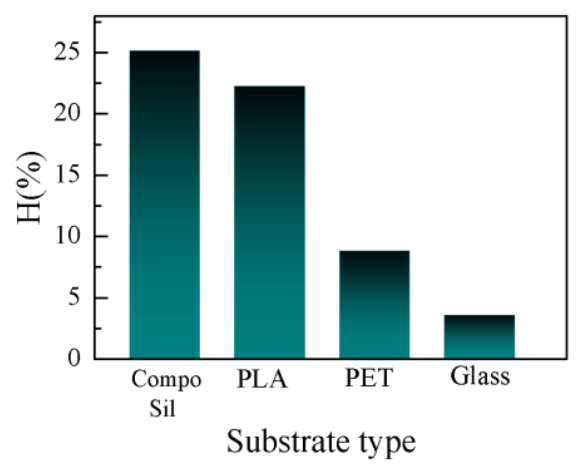

Figure 4. Percentage of hysteresis, H calculated with Equation (1) for Compo-Sil, PLA, PET and glass substrates with deposited Au:Pd/PVA-Ac24 at low and high relative humidity.

The smallest $\mathrm{H}$-value for flexible substrates of $8.9 \%$ is achieved for PVA-Ac24 deposited on PET which is almost three times smaller compared to samples PLA (22.3\%) and Compo-Sil (25.2\%).

\section{Conclusions}

The successful humidity sensing application of thin films of poly(vinyl alcohol-co-vinyl acetal) with acetal content $24 \%$ deposited on PET, PLA and Compo-Sil flexible substrates is demonstrated. All types of substrates used are shown to be suitable for deposition of metal (Au:Pd) sublayer and for polymers deposition via spin-coating. The best sensor characteristics are obtained for PVA-Ac24 films deposited on PET substrate-highest transmittance change and smallest hysteresis value. PET substrate with $\mathrm{Au}: \mathrm{Pd} /$ polymer deposited on top has the smoothest surface and even distribution of the thickness with no visible cracks and defects. Results obtained for this substrate are similar to the results for glass sample with $\mathrm{Au}: \mathrm{Pd} /$ polymer thus taking advantage of the flexibility of the substrate but retaining the excellent optical properties of the glass which gives new opportunities for their potential feature use as in situ optical flexible/wearable sensors for humidity in the surrounding environment.

Author Contributions: Conceptualization, T.B., D.C. and K.L.; methodology, T.B., D.C., and K.L.; software, T.B. and K.L.; validation, T.B., D.C., and K.L.; formal analysis, S.B., S.I. and K.L.; investigation, K.L. and S.B.; resources, D.C. and T.B.; data curation, K.L., D.C. and T.B.; writing-original draft preparation, K.L.; writingreview and editing, T.B., D.C. and K.L.; visualization, T.B. and K.L.; supervision, T.B. and D.C.; project administration, T.B. and K.L.; All authors have read and agreed to the published version of the manuscript

Funding: This research received partial funding from project DN08-15/2016 (Bulgarian National Science Fund).

Acknowledgments: K. Lazarova and S. Bozhilova acknowledge the National Scientific Program for young scientists and postdoctoral fellows, funded by the Bulgarian Ministry of Education and Science (MES) with DCM 577/2018 and 271/2019. Research equipment of the distributed research infrastructure INFRAMAT (part of the Bulgarian National roadmap for research infrastructure) supported by the Bulgarian Ministry of Education and Science under contract D01-284/17.12.2019 was used in this investigation.

Conflicts of Interest: The authors declare no conflict of interest.

\section{References}

1. Kolpakov, S.A.; Gordon, N.T.; Mou, C.; Zhou, K. Toward a new generation of photonic humidity sensors. Sensors 2014, 14, 3986-4013.

2. Sikarwar, S.; Yadav, B.C. Opto-electronic humidity sensor: A review. Sens. Actuators A 2015, 233, 54-70.

3. Xuan, X.; Yoon, H.S.; Park, J.Y. A wearable electrochemical glucose sensor based on simple and low-cost fabrication supported micro-patterned reduced graphene oxide nanocomposite electrode on flexible substrate. Biosens. Bioelectron. 2018, 109, 75-82. 
4. Xu, H.; Xiang, J.X.; Lu, Y.F.; Zhang, M.K.; Li, J.J.; Gao, B.B.; Zhao, Y.J.; Gu, Z.Z. Multifunctional Wearable Sensing Devices Based on Functionalized Graphene Films for Simultaneous Monitoring of Physiological Signals and Volatile Organic Compound Biomarkers. ACS Appl. Mater. Interfaces. 2018, 10, 11785-11793.

5. Sekine, T.; Sugano, R.; Tashiro, T.; Sato, J.; Takeda, Y.; Matsui, H.; Kumaki, D.; Dos Santos, F.D.; Miyabo, A.; Tokito, S. Fully Printed Wearable Vital Sensor for Human Pulse Rate Monitoring using Ferroelectric Polymer. Sci. Rep. 2018, 8, 4442.

6. Liu, Q.; Liu, Y.; Wu, F.; Cao, X.; Li, Z.; Alharbi, M.; Abbas, A.N.; Amer, M.R.; Zhou, C. Highly Sensitive and Wearable $\mathrm{In}_{2} \mathrm{O}_{3}$ Nanoribbon Transistor Biosensors with Integrated On-Chip Gate for Glucose Monitoring in Body Fluids. ACS Nano 2018, 12, 1170-1178.

7. Costa, J.C.; Spina, F.; Lugoda, P.; Garcia-Garcia, L.; Roggen, D.; Münzenrieder, N. Flexible Sensors -From Materials to Applications. Technologies 2019, 7, 35.

8. Xu, K.; Lu, Y.; Takei, K. Multifunctional Skin-Inspired Flexible Sensor Systems for Wearable Electronics. Adv. Mater. Technol. 2019, 4, 1800628.

9. Zhang, J.; Zhong, J.; Fang, Y.; Wang, J.; Huang, G.; Cui, X.; Mei, Y. Roll up polymer/oxide/polymer nanomembranes as a hybrid optical microcavity for humidity sensing. Nanoscale 2014, 6, 13646-13650.

10. Naydenova, I.; Jallapuram, R.; Toal, V.; Martin, S. A visual indication of environmental humidity using a color changing hologram recorded in a self-developing photopolymer. Appl. Phys. Lett. 2008, 92, 031109.

11. Naydenova, I.; Grand, J.; Mikulchyk, T.; Martin, S.; Toal, V.; Georgieva, V.; Thomas, S.; Mintova, S. Hybrid Sensors Fabricated by Inkjet Printing and Holographic Patterning. Chem. Mater. 2015, 27, 6097-6101.

12. Wei, M.; Gao, Y.; Li, X.; Serpe, M.J. Stimuli responsive polymers and their applications. Polym. Chem. 2017, 8, 127-143.

13. Lazarova, K.; Bozhilova, S.; Christova, D.; Babeva, T. Poly(vinyl alcohol)-based thin films for optical humidity sensing. J. Phys. Conf. Ser. 2020, 1492.

14. Lazarova, K.; Bozhilova, S.; Ivanova, S.; Christova, D.; Babeva, T. The Influence of Annealing on Optical and Humidity Sensing Properties of Poly(Vinyl Alcohol-co-Vinyl Acetal) Thin Films. Proceedings 2020, 42, 16.

15. Christova, D.; Ivanova, S.; Ivanova, G. Water-soluble temperature-responsive poly(viny1 alcohol-co-vinyl acetal)s. Polym. Bull. 2003, 50, 367-372.

16. Lazarova, K.; Vasileva, M.; Marinov, G.; Babeva, T. Optical characterization of sol-gel derived $\mathrm{Nb}_{2} \mathrm{O}_{5}$ thin films. Opt. Laser Technol. 2014, 58, 114-118.

17. Lazarova, K.; Awala, H.; Thomas, S.; Vasileva, M.; Mintova, S.; Babeva, T. Vapor responsive onedimensional photonic crystals from zeolite nanoparticles and metal oxide films for optical sensing. Sensors 2014, 14, 12207-12218.

18. Lazarova, K.; Bozhilova, S.; Novakov, Ch.; Christova, D.; Babeva, T. Amphiphilic Poly(vinyl Alcohol) Copolymers Designed for Optical Sensor Applications-Synthesis and Properties. Coatings 2020, 10, 460.

Publisher's Note: MDPI stays neutral with regard to jurisdictional claims in published maps and institutional affiliations.

2020 by the authors. Submitted for possible open access publication under the terms and conditions of the Creative Commons Attribution (CC BY) license (http://creativecommons.org/licenses/by/4.0/). 